\title{
Ecuaciones de Bloch Ópticas en Sistemas Complejos con Acoplamiento Intramolecular
}

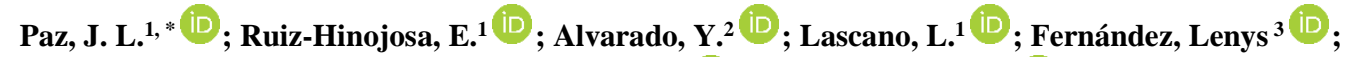

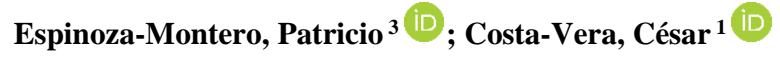 \\ ${ }^{1}$ Departamento de Física, Escuela Politécnica Nacional, Quito, Ecuador \\ ${ }^{2}$ Laboratorio de Caracterización Molecular y Biomolecular (LCMB), Centro de Investigación y Tecnología de Materiales \\ (CITeMA), Instituto Venezolano de Investigaciones Científicas (IVIC) Región Zulia, República Bolivariana de Venezuela; \\ ${ }^{3}$ Escuela de Química, Facultad de Ciencias Exactas y Naturales, Pontificia Universidad Católica del Ecuador, Quito, Ecuador
}

\begin{abstract}
Resumen: Proponemos modificaciones de las ecuaciones de Bloch ópticas convencionales para un sistema molecular, cuando consideramos los efectos de acoplamiento intramolecular. Modelamos la molécula aislada como curvas de energía de Born-Oppenheimer que consisten en dos estados electrónicos cruzados descritos como potenciales armónicos, con los mínimos desplazados en coordenadas nucleares y energía. Consideramos dos estados vibracionales y una perturbación, que puede surgir de una correlación residual electrón-electrón y/o términos de acoplamiento spin-órbita en el Hamiltoniano del sistema, causando la separación de las dos curvas según la regla del cruce evitado. Las ecuaciones extendidas de Bloch ópticas permiten establecer la dinámica de un sistema molecular de estados adiabáticos sujeto a la interacción de un reservorio térmico e interactuando con campos electromagnéticos. En las ecuaciones se observa que haciendo nulo el factor de acoplamiento intramolecular, se recuperan las ecuaciones de Bloch ópticas convencionales para un sistema molecular con potenciales armónicos cuyos mínimos se encuentran exactamente en la misma coordenada nuclear sujeto a la aproximación Born-Oppenheimer.
\end{abstract}

Palabras clave: acoplamiento intramolecular, ecuaciones ópticas de Bloch, adiabáticos, Born-Oppenheimer

\section{On the Optical Bloch Equations in Complex Systems with Intramolecular Coupling}

\begin{abstract}
We propose modifications of the conventional optical Bloch equations for a complex system, when we consider intermolecular coupling effects. We model the isolated molecule as Born-Oppenheimer energy curves consisting of two-electronic states described as harmonic potentials, with minima displaced in nuclear coordinates and energy. We consider two-vibrational states and we include non-adiabatic effects for this two states model. Inclusion of a residual perturbation $\tilde{H}$, which may arise from a residual electron-electron correlation and/or spinorbit coupling terms in the Hamiltonian of the system, may couple the above electronic states, causing the separation of the two curves according to the avoided-crossing rule. The extended equations of optical Bloch allow to establish the dynamics of a molecular system of adiabatic states subject to the interaction of a thermal reservoir and interacting with electromagnetic fields. The equations show that by making the intramolecular coupling factor zero, the conventional optical Bloch equations are recovered for a molecular system with harmonic potentials whose minima are found in exactly the same nuclear coordinate subject to the Born-Oppenheimer approximation.
\end{abstract}

Keywords: intramolecular coupling, optical Bloch equations, non-adiabatic effects, Born-Oppenheimer

\section{INTRODUCCIÓN}

El modelo de dos estados para la descripción de los átomos y moléculas que interactúan con un campo electromagnético clásico ha demostrado ser útil en la óptica no lineal. En una molécula, tales estados son generalmente pensados como estados vibracionales que pertenecen a una o dos superficies de energías potenciales. En este trabajo, consideramos sólo el segundo caso, pero tenemos en cuenta la posibilidad de un acoplamiento intramolecular directo de estado a estado. Este tipo de modelo que consiste en dos 
curvas electrónicas, ya sea degeneradas o no degeneradas para una pequeña región de valor de coordenadas nucleares, se ha aplicado al tratamiento de los efectos Jahn-Teller y pseudo-Jahn-Teller en las moléculas (García-Sucre y col., 1970; Slivnev y col.,2020) y al acoplamiento vibrónico que se produce para los estados excitados degenerados de los dímeros (Witkowsky y col., 1960). Nuestro modelo consiste en dos estados electrónicos cuyas curvas de energía potencial se describen como osciladores armónicos desplazados en energía y coordenada nuclear. Consideramos la presencia de estados electrónicos moleculares con momentos dipolares permanentes no nulos. Nuestro sistema efectivo de dos niveles de estados vibratorios se describe en la Figura 1.

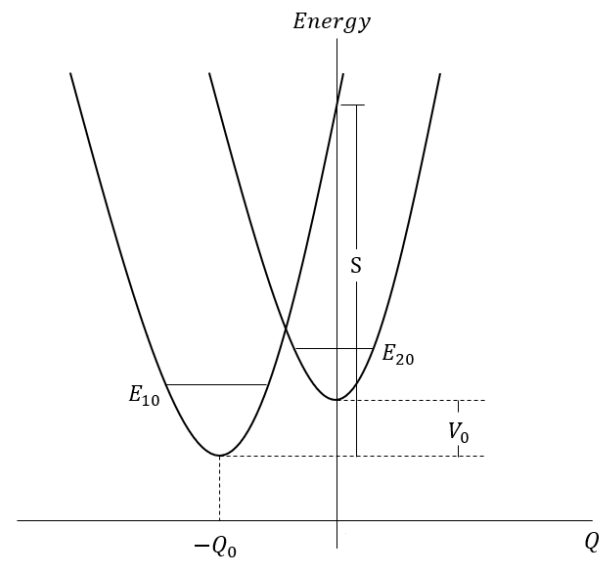

Figura 1. Representación diabática. Los mínimos de los dos potenciales están desplazados verticalmente en energía por $V_{0}$ y horizontalmente por

$$
\text { la distancia } Q_{0} \text {. }
$$

Esta descripción de los estados electrónicos entrecruzados corresponde a la llamada representación diabática. También se puede incluir una perturbación residual $\tilde{H}$, acoplando estos estados electrónicos. Las curvas anteriores pueden separarse según la regla de cruce evitado (Figura 2).

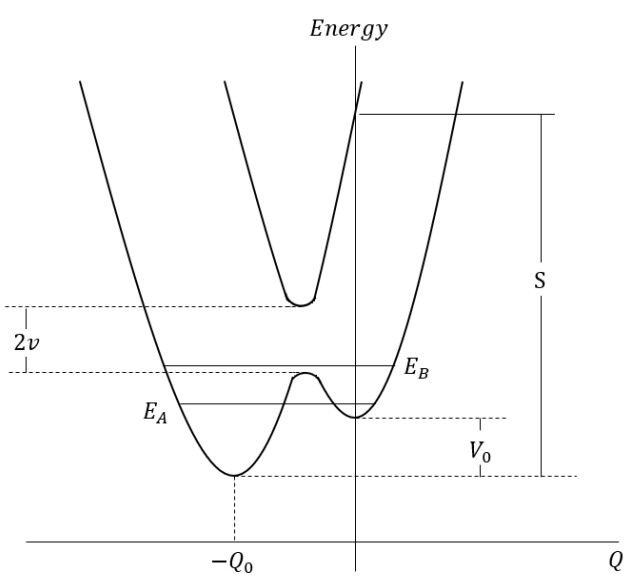

Figura. 2. Representación adiabática. La perturbación residual que da lugar a la separación de las dos curvas armónicas se representa de acuerdo con una regla de cruce evitado.
Estos dos nuevos estados $\left\{E_{A}, E_{B}\right\}$ del Hamiltoniano electrónico pertenecen ahora a la llamada representación adiabática y su forma depende del valor del elemento matriz no diagonal de $\tilde{H} ; E_{A}$ y $E_{B}$ denotan las energías vibrónicas resultantes después de la inclusión del acoplamiento $\tilde{H}$. El acoplamiento intramolecular es una herramienta fundamental para explicar las transiciones entre los estados electrónicos en los espectros de absorción y emisión que se suponía que estaban prohibidas por la simetría. Las descripciones teóricas de los procesos fotofísicos y fotoquímicos en la literatura implican el tratamiento de dos o más interacciones electrónicovibracionales (Thoss y col., 2000). El acoplamiento vibrónico, resultante del acoplamiento entre los movimientos nucleares y electrónicos en una molécula es de gran importancia para algunos procesos físico-químicos, por ejemplo, en el estudio de las tasas de relajación de la conversión interna (Mebel y col., 1997), en el estudio de las probabilidades de transición débiles prohibidas por la simetría en los procesos de absorción y emisión, en la espectroscopia de femtosegundos (Domcke y Stock, 1997), en el estudio de la forma de la banda de absorción óptica de los dímeros (Pakhomov y col., 1995) y en el estudio de las resonancias en los procesos de dispersión (Charutz y col., 1997).

Nuestro sistema molecular ahora queda representado por un sistema de dos niveles no convencional, en el sentido que se lo describe en términos del acoplamiento intramolecular a través de sus estados adiabáticos. Considerando ahora este nuevo esquema, la dinámica temporal de este sistema en presencia de un baño térmico e interactuando con los campos electromagnéticos clásicos, se trata a través de las ecuaciones ópticas convencionales de Bloch, haciendo uso del formalismo de la matriz de densidad y de la ecuación de Liouville y Von Newmann. En este trabajo, proponemos una extensión de las ecuaciones ópticas convencionales de Bloch para sistemas complejos en presencia de acoplamientos intramoleculares.

\subsection{REPRESENTACIÓN DIABÁTICA}

La inclusión de la interacción entre el movimiento nuclear y electrónico en el cálculo de los valores y vectores propios del sistema molecular a través de $H_{T} \Psi_{T}(r, Q)=E_{T} \Psi_{T}(r, Q) \quad$ requiere, según la formulación general del modelo de acoplamiento intramolecular de Herzberg y Teller, poder expresar las funciones de onda electrónicas con una dependencia paramétrica en el espacio de las coordenadas nucleares Q . Estrictamente el término acoplamiento intramolecular se refiere a la corrección de las funciones de onda electrónicas de Born-Oppenheimer $\Psi_{T}(r, Q)=\psi(r) \chi(Q)$ a fin de obtener información sobre la interacción entre los movimientos electrónicos y nucleares del sistema (Zhao $y$ col., 2020). Con este fin, el término de acoplamiento $U(r, Q)$ se desarrolla en serie de Taylor para los 
pequeños movimientos del núcleo alrededor de la configuración de referencia previamente elegida $Q_{0}$. Para simplificar,

obtenemos

$U(r, Q)=U\left(r, Q_{0}\right)+\Delta U(r, Q) . \quad$ En $\quad$ esta representación, las coordenadas nucleares (en el espacio) se consideran fijas, introducidas en la función de onda $\psi_{\mathrm{k}}$ como parámetro. El movimiento de los electrones se obtiene con: $\quad\left[H_{\text {elect }}+U(r, Q)\right] \psi_{k}(r, Q)=\varepsilon_{k} \psi_{k}(r, Q)$. Ahora, la función de onda total se desarrolla como $\Psi(r, Q)=\sum_{k} \chi_{k}(Q) \psi_{k}(r, Q)$, donde $\chi_{k}(Q)$ es una función de onda vibracional en la representación diabática, calculada según

$\left[T_{N}(Q)+\varepsilon(Q)\right] \chi_{k}(Q)+\sum_{k} W_{k n}(Q) \chi_{n}(Q)$

$=E_{T} \chi_{k}(Q)$, donde $W_{k n}$ es el operador de acoplamiento no diabático.

\subsection{REPRESENTACIÓN ADIABÁTICA}

La función de onda electrónica se encuentra para una configuración nuclear específica $Q_{0}$ que no necesariamente se refiere a la posición real del núcleo en el espacio, dada por $\left[H_{\text {elect }}+U\left(r, Q_{0}\right)\right] \psi_{i}^{0}\left(r, Q_{0}\right)=\varepsilon_{k} \psi_{i}^{0}\left(r, Q_{0}\right)$, donde $\psi_{i}^{0}\left(r, Q_{0}\right)$ son las funciones de onda electrónica diabáticas. La función de onda total, es $\Psi(r, Q)=\sum_{i} \chi_{i}^{0}(Q) \psi_{i}^{0}\left(r, Q_{0}\right)$ y finalmente,

$$
\begin{aligned}
C_{i j}(Q) & =\frac{\left\langle\psi_{i}^{0}\left(r, Q_{0}\right)|\Delta U(r, Q)| \psi_{j}^{0}\left(r, Q_{0}\right)\right\rangle}{\varepsilon_{j}^{0}+\varepsilon_{i}^{0}} \\
& +\sum_{k \neq j} \frac{\left\langle\psi_{i}^{0}\left(r, Q_{0}\right)|\Delta U(r, Q)| \psi_{k}^{0}\left(r, Q_{0}\right)\right\rangle\left\langle\psi_{k}^{0}\left(r, Q_{0}\right)|\Delta U(r, Q)| \psi_{j}^{0}\left(r, Q_{0}\right)\right\rangle}{\left(\varepsilon_{j}^{0}-\varepsilon_{i}^{0}\right)\left(\varepsilon_{j}^{0}-\varepsilon_{k}^{0}\right)}+\ldots
\end{aligned}
$$

donde $\left\langle\psi_{j}(r, Q)|\Delta U(r, Q)| \psi_{k}(r, Q)\right\rangle$ genera la superficie de energía potencial adiabática de los núcleos en el campo generado por los electrones. Por lo tanto, el valor de los elementos $\sum_{k \neq j}\left\langle\psi_{j}(r, Q)|\Delta \mathrm{U}(r, Q)| \psi_{k}(r, Q)\right\rangle \chi_{k}(Q)=0$ mide el efecto del acoplamiento intramolecular (Mita y col., 2020). Finalmente, utilizando la teoría de perturbaciones de Rayleigh-Schrödinger, se obtiene la función de onda electrónica corregida:

$$
\begin{aligned}
& \begin{array}{l}
\psi_{i}(r, Q)=\psi_{i}^{0}\left(r, Q_{0}\right) \\
+\sum_{i \neq j} C_{i j}(Q) \psi_{i}^{0}\left(r, Q_{0}\right)
\end{array} \\
& \Psi_{T}(r, Q)=\left[\psi_{i}^{0}\left(r, Q_{0}\right)\right. \\
& \left.+\sum_{i \neq j} C_{i j}(Q) \psi_{i}^{0}\left(r, Q_{0}\right)\right] \chi_{j}(Q)
\end{aligned}
$$

donde:
Las condiciones para el cruce de dos curvas de potencial adiabático de la misma simetría en un modelo de acoplamiento de dos estados, requiere $\Delta U_{i i}(r, Q)=\Delta U_{j j}(r, Q) \quad$ y $\quad \Delta U_{i j}(r, Q)=0 . \quad \mathrm{El}$ cruce de superficies de energía potencial se asocia con los problemas de Jahn-Teller, donde las simetrías de estado generan intersecciones cónicas. La inestabilidad de algunas configuraciones nucleares produce una distorsión instantánea de su estructura que elimina la degeneración.

\section{MODELO DE ACOPLAMIENTO INTRAMOLECULAR}

El Hamiltoniano asociado al sistema molecular en presencia de acoplamiento intramolecular se define como $H=H_{0}+H(r)$, donde $H_{0}$ representa los dos osciladores armónicos desacoplados entre sí (sistema molecular de dos niveles con estados vibracionales sin acoplamiento intramolecular), y $H(r)$ corresponde a un término residual de perturbación (dependiendo sólo de las coordenadas electrónicas) que induce el acoplamiento 
intramolecular. Las funciones propias de $H_{0}$ son $\left|\psi_{1}\right\rangle$ y $\left|\psi_{2}\right\rangle$, donde y $H_{0}\left|\psi_{2}\right\rangle=E_{2 k}\left|\psi_{2}\right\rangle$, con estados electrónicos $\{1,2\}$ y estados vibracionales $\{j, k\}$. Para la inclusión de $H(r)$ en el Hamiltoniano total, tenemos $H\left|\psi_{A}\right\rangle=E_{A}\left|\psi_{A}\right\rangle \quad$ y $\quad H\left|\psi_{B}\right\rangle=E_{B}\left|\psi_{B}\right\rangle$. En la aproximación de Born-Oppenheimer $\left|\psi_{A}\right\rangle=\left|\chi_{1 j} \psi_{1}\right\rangle \mathrm{y}$ $\left|\psi_{B}\right\rangle=\left|\chi_{2 k} \psi_{2}\right\rangle$. El acoplamiento intramolecular está representado por los elementos no diagonales del operador residual:

$$
\begin{aligned}
\left\langle\chi_{1 j} \psi_{1}|H| \chi_{2 k} \psi_{2}\right\rangle & =W_{12} \\
& =v\left\langle\chi_{1 j} \mid \chi_{2 k}\right\rangle
\end{aligned}
$$

donde $v=\left\langle\psi_{1}|H| \psi_{2}\right\rangle$, con $v$ como el coeficiente o parámetro de acoplamiento y $\left\langle\chi_{1 j} \mid \chi_{2 k}\right\rangle$ la integral de solapamiento de los estados vibracionales. Los valores de energía y las correspondientes funciones propias pueden ser escritas de la forma:

$$
\begin{aligned}
& E_{B}=\frac{1}{2}\left\{\left(E_{1 j}+E_{2 k}\right)-\left[\left(E_{1 j}-E_{2 k}\right)^{2}\right.\right. \\
& \left.\left.+4 v^{2}\left|\left\langle\chi_{1 j} \mid \chi_{2 k}\right\rangle\right|^{2}\right]^{1 / 2}\right\} \\
& E_{A}=\frac{1}{2}\left\{\left(E_{1 j}+E_{2 k}\right)+\right. \\
& \left.\left[\left(E_{1 j}-E_{2 k}\right)^{2}+4 v^{2}\left|\left\langle\chi_{1 j} \mid \chi_{2 k}\right\rangle\right|^{2}\right]^{1 / 2}\right\}
\end{aligned}
$$

$$
\begin{aligned}
& \left|\Psi_{B}\right\rangle=\left|\Psi_{B}(r, Q)\right\rangle \\
& =C_{1 j}^{B}\left[\psi_{1}(r ; Q) \chi_{1 j}(Q)\right. \\
& \left.+A_{j k}^{B} \psi_{2}(r ; Q) \chi_{2 k}(Q)\right] \\
& \left|\Psi_{A}\right\rangle=\left|\Psi_{A}(r, Q)\right\rangle \\
& =C_{1 j}^{A}\left[\psi_{1}(r ; Q) \chi_{1 j}(Q)\right. \\
& \left.+A_{j k}^{A} \psi_{2}(r ; Q) \chi_{2 k}(Q)\right]
\end{aligned}
$$

donde r y Q corresponden a las coordenadas electrónicas y nucleares, respectivamente, y donde se encuentran las siguientes cantidades:

$$
\begin{aligned}
& E_{1 j}=\left(j+\frac{1}{2}\right) \hbar \omega_{0}, E_{2 k}=\left(k+\frac{1}{2}\right) \hbar \omega_{0}+ \\
& V_{0} ; C_{1 j}^{A}=\left(1+A_{j k}^{A}\right)^{-1 / 2}, C_{1 j}^{B}=\left(1+A_{j k}^{B}\right)^{-1 / 2} \\
& \operatorname{con} A_{j k}^{A}=\frac{E_{1 j}-E_{A}}{v\left\langle\chi_{1 j} \mid \chi_{2 k}\right\rangle} \text { y } A_{j k}^{B}=\frac{E_{1 j}-E_{B}}{v\left\langle\chi_{1 j} \mid \chi_{2 k}\right\rangle} .
\end{aligned}
$$

Normalmente en los cálculos se utilizan algunos criterios que definen la intensidad de acoplamiento: débil $\left(v \ll^{S \hbar \omega_{0} / 4}\right)$, intermedio $\left(v \approx S \hbar \omega_{0} / 4\right)$ y fuerte $\left(v \gg S \hbar \omega_{0} / 4\right)$, donde $S=\left(m \omega_{0} / \hbar\right) Q_{0}^{2}$, m es la masa asociada al modo vibracional descrito por la coordenada nuclear $\mathrm{Q}$ y donde $Q_{0}$ representa la separación de coordenadas de los dos mínimos de potenciales electrónicos La frecuencia de Bohr $\omega_{0}$ viene dada por $\omega_{0}=\left(E_{2 k}-E_{1 j}\right) / \hbar$. Ahora es necesario hacer una descripción de los momentos dipolares permanentes y de transición en los estados adiabáticos para la extensión de las ecuaciones ópticas convencionales de Bloch.

\section{MOMENTOS DIPOLARES PERMANENTES Y DE TRANSICIÓN EN LOS ESTADOS ADIABÁTICOS.}

En nuestro modelo, los momentos dipolares permanentes y de transición entre los estados $\left|\psi_{A}\right\rangle$ y $\left|\psi_{B}\right\rangle$ se pueden escribir como:

$$
\begin{aligned}
& \hat{\mu}_{A B}=-C_{1 j}^{A} C_{1 j}^{B}\left[A_{j k}^{A} A_{j k}^{B} m_{22}(0)+m_{11}\left(-Q_{0}\right)\right] \\
& -C_{1 j}^{A} C_{1 j}^{B} m_{12}\left(-Q_{0}\right)\left\langle\chi_{1 j} \mid \chi_{2 k}\right\rangle\left(A_{j k}^{B}+A_{j k}^{A}\right) \\
& \hat{\mu}_{r r}=\left(C_{1 j}^{r}\right)^{2}\left[\left(A_{j k}^{r}\right)^{2} m_{22}(0)+m_{11}\left(-Q_{0}\right)\right] \\
& -2\left(C_{1 j}^{r}\right)^{2} m_{12}\left(-Q_{0}\right)\left\langle\chi_{1 j} \mid \chi_{2 k}\right\rangle\left(A_{j k}^{r}\right)
\end{aligned}
$$

Con $r=A, B$, y donde $m_{11}$ y $m_{22}$ representan los momentos dipolares permanentes asociados a los estados desacoplados $\left|\psi_{1}\right\rangle$ y $\left|\psi_{2}\right\rangle$. En este caso: 


$$
m_{i j}(Q)=\int \psi_{i}^{*}(r ; Q) \hat{m} \psi_{j}(r ; Q) d^{3} r
$$

Donde $\mathrm{m}$ es el operador de momento dipolar electrónico, para valores $i, j=1,2$. El factor de solapamiento vibracional $\left\langle\chi_{1 j} \mid \chi_{2 k}\right\rangle$ puede ser evaluado de acuerdo con la fórmula de Pekarian (Di Bartolo y Goldberg, 1980):

$$
\begin{aligned}
& \left\langle\chi_{1 j} \mid \chi_{2 k}\right\rangle=\frac{(-1)^{k}}{(k !)^{1 / 2}} S^{k / 2} \exp \left(-\frac{S}{4}\right) 2^{-k / 2} \\
& \text { para } j=0
\end{aligned}
$$

Tenemos $m_{11}$ y $m_{22}$ evaluado en $-Q_{0}$ y 0 , respectivamente, mientras que las Ecs. (6) y (7), y dado el principio de Franck-Condon $m_{12}$ se evalúa en $-Q_{0}$. Esto equivale a evaluar los momentos permanentes y de transición en los estados desacoplados en la geometría de equilibrio. Sin embargo, estas geometrías se mezclan en la evaluación de los momentos en los estados acoplados. En las ecuaciones (6-8), primero observamos que, aun cuando los estados desacoplados tienen un momento dipolar permanente nulo $m_{11}=m_{22}=0$, el acoplamiento induce un momento no nulo en los estados acoplados proporcionados $m_{12} \neq 0$. En segundo lugar, cuando $m_{11}$, $m_{22}$ son diferentes de cero, un caso importante en este trabajo, las contribuciones de $m_{11}$ y $m_{22}$ a $\mu_{a a}(a=A, B)$ pueden diferir sustancialmente debido tanto al factor de acoplamiento como al de Franck-Condon, mientras que la única manera en que los momentos del dipolo permanente $m_{11}$ y $m_{22}$ y contribuyen a $\mu_{A B}$ es a través de la diferencia $m_{11}-m_{22}$. En particular, cuando $m_{11}=m_{22} \neq 0$, estos momentos del dipolo permanente contribuyen a $\mu_{a a}(a=A, B)$ haciendo $\mu_{b b}$ diferente de $\mu_{A A}$ lo cual tiene consecuencias importantes para las respuestas no lineales. En tercer lugar, el interesante efecto del momento de transición $\mu_{A B}$ que va en sentido inverso al de los momentos del dipolo permanente mencionados anteriormente, según el cual $\mu_{A B}$ puede desaparecer como efecto del acoplamiento intramolecular a pesar de que el momento del dipolo de transición en el estado desacoplado $m_{12} \neq 0$. Esto es, $v=-m_{12}\left(E_{1 j}-E_{2 k}\right) /\left(m_{22}-m_{11}\right)$ y considerando esta expresión para el degenerado, las condiciones para el efecto mencionado son: i) los dos estados mezclados por el acoplamiento intramolecular deben ser no degenerados; y ii) los signos de $m_{12}\left(E_{1 j}-E_{2 k}\right)$ y $m_{22}-m_{11}$ deben ser opuestos. En cuarto lugar, el caso degenerado, $E_{1 j}=E_{2 k}$ merece una atención especial. En este caso $\mu_{A B}$ y $\mu_{a a}(a=A, B)$ se convierten

$$
\begin{aligned}
& \mu_{A B}=\frac{1}{2}\left[m_{11}\left(-Q_{0}\right)-m_{22}(0)\right] \\
& \mu_{a a}=\frac{1}{2}\left[m_{11}\left(-Q_{0}\right)+m_{22}(0)\right] \\
& \pm\left[\left\langle\chi_{1 j} \mid \chi_{2 k}\right\rangle m_{12}\left(-Q_{0}\right)\right]
\end{aligned}
$$

empleando el signo negativo en el estado $|A\rangle$ y el signo positivo para el estado $|B\rangle$.

Los momentos de transición y de dipolo permanente son ahora independientes del acoplamiento $v$, además $\mu_{A B}$, no contiene ninguna contribución de $m_{12}$.

Estos resultados no se mantendrán exactamente para una función de onda más general:

$$
\Psi(r, Q)=C_{i j} \psi_{1}(r ; Q) \chi_{1 j}(Q)+C_{2 k} \psi_{2}(r ; Q) \chi_{2 k}(Q)
$$

pero un argumento perturbativo indica que incluso para una mezcla más general, las ecuaciones. (10) y (11) se mantendrán aproximadamente si hay una contribución dominante de cualquiera de los dos estados degenerados.

\section{ECUACIONES ÓPTICAS DE BLOCH EN ESTADOS ADIABÁTICOS}

Es importante destacar que nuestro sistema molecular aislado es representado en la base adiabática correspondiente a los estados $|A\rangle$ y $|B\rangle$. Para estudiar la extensión de las ecuaciones de Bloch ópticas convencionales, ahora en la base adiabática, este sistema que hemos descrito estará sujeto a la interacción por la presencia del solvente, y a una interacción radiativa por efecto de los campos electromagnéticos. En orden de describir la evolución temporal del sistema, hacemos uso de la ecuación de Liouville dada por $i \hbar \partial_{t} \rho=[H, \rho]$, donde $\rho(t)$ es la matriz densidad reducida, mientras $\mathrm{H}$ es el Hamiltoniano total, el cual describe al soluto, solvente, capos electromagnéticos y todas las interacciones entre ellos, dado por $\mathrm{H}=\mathrm{H}_{S}+$ $H_{F}+H_{R}+H_{S F}+H_{S R}+H_{F R}$. Ahora $H_{S}$ corresponderá al Hamiltoniano del sistema aislado sujeto al efecto intramolecular y descrito por $H_{S}=H_{0}+H(r)$, 
donde $H_{0}$ corresponderá al Hamiltoniano en la base diabática, mientras que $H(r)$ representará la perturbación relativa al acoplamiento intramolecular; $H_{R}$ representa el Hamiltoniano del reservorio térmico, el cual es despreciado en el presente estudio; $H_{F}$ corresponde al Hamiltoniano del campo electromagnético, el cual no tomamos en cuenta por la consideración de la aproximación semiclásica. Para completar la descripción espectroscópica de interacción radiación-materia, consideraremos $H_{S F}$ para representar la interacción entre el soluto (sistema activo con acoplamiento intramolecular) y el campo, el cual describimos de acuerdo con la aproximación de tipo dipolar; $H_{S R}$ representa la interacción soluto-solvente descrita en términos de los mecanismos de relajación, y finalmente $H_{F R}$ que representa la interacción entre el campo de radiación y el solvente. Esta última interacción no la tomamos en cuenta, al considerar que la frecuencia del campo perturbador no es sintonizable o resonante a la frecuencia de respuesta del reservorio térmico. Normalmente se dice en este último que el solvente es transparente a la radiación, dado que no hay absorción. En orden de obtener la evolución temporal del sistema global, debemos considerar previamente algunas aproximaciones, algo más fundamentales. Suponemos un reservorio térmico con muchísimos grados de libertad, de forma que la interacción con el soluto la energía sea disipada rápidamente, haciendo que el reservorio permanezca en el régimen de equilibrio térmico. Este hecho se traduce en que $\rho(t)_{S-R}=\rho(t)_{S} \bigotimes \rho(0)_{R}$, además de considerar que el sistema en su interacción con el campo electromagnético pierde su memoria y evoluciona a partir del momento que interacciona con el campo. Esto último corresponde a la aproximación Markoviana. Una revisión más detallada de las restantes aproximaciones fundamentales que tienen lugar en este conjunto de interacciones puede ser revisada en la referencia (Blum, 1981). Considerando entonces las interacciones mencionadas, y haciendo uso de la teoría cuántica de relajación, la evolución temporal del elemento de matriz densidad reducida, sólo para el subsistema, queda expresada de la forma:

$$
\begin{aligned}
& \left(\partial_{t} \rho_{S}\right)_{m^{\prime} m}=-i \omega_{m^{\prime} m}\left(\rho_{S}\right)_{m^{\prime} m} \\
& -\frac{1}{\hbar}\left\langle m^{\prime}\left|\left[H_{S F}, \rho_{S}\right]\right| m\right\rangle+\sum_{n n^{\prime}} R_{m^{\prime} m n^{\prime} n}\left(\rho_{S}\right)_{n^{\prime} n}
\end{aligned}
$$

donde $R_{m m^{\prime} m n^{\prime}}$ es la matriz de relajación, cuya forma exacta puede ser vista en la referencia indicada, pero su deducción completa no es colocada en el presente trabajo. Nótese que la expresión (12) obedece a la evolución temporal de una parte reducida del sistema, adjudicada al subsistema, distinto a lo que ocurre en la ecuación de Liouville, la cual permite la evolución sólo de sistemas completos. Las ecs. (6) y (7) constituyen el punto central de este trabajo. En ellas se incluyen los efectos de la base acoplada (acoplamiento intramolecular) sobre el sistema de dos niveles, el ensanchamiento homogéneo de la línea espectral y la interacción del sistema con el reservorio. En este trabajo, hemos considerado que la presencia del reservorio térmico no modifica parámetros fundamentales en la respuesta del soluto. El primer término de la ec.(12) describe el sistema molecular acoplado con su frecuencia de resonancia $\omega_{m^{\prime} m}=\omega_{0}$ (frecuencia de Bohr en la base adiabática); el segundo término es relativo a la interacción de carácter dipolar eléctrico entre el sistema y el campo de radiación, mientras que el último término, corresponde al mecanismo de relajación del sistema introducido en forma fenomenológico y representado por la matriz de Bloembergen del tipo $R_{m^{\prime} m n^{\prime} n}$ (Blum, 1981). Con todas estas consideraciones en mente, la dinámica del sistema es obtenida resolviendo las siguientes ecuaciones de Bloch ópticas para los estados adiabáticos:

$$
\partial_{t} \rho=M(t) \rho+R
$$

donde

$$
\begin{aligned}
& \rho=\left(\begin{array}{c}
\rho_{A B} \\
\rho_{B A} \\
\rho_{D}
\end{array}\right) \\
& M(t)=\left(\begin{array}{ccc}
0 & f(v) & \frac{i}{\hbar} \mu_{A B} E \\
f^{*}(v) & 0 & -\frac{i}{\hbar} \mu_{B A} E \\
-\frac{2 i}{\hbar} \mu_{A B} E & \frac{2 i}{\hbar} \mu_{B A} E & -\frac{1}{T_{1}}
\end{array}\right) \\
& f(v)=i \omega_{0}-\frac{1}{T_{2}}-\frac{i}{\hbar} d E \\
& R=\left(\begin{array}{c}
0 \\
0 \\
\frac{1}{T_{1}} \rho_{D}^{(0)}
\end{array}\right) ; d=\mu_{B B}-\mu_{A A}
\end{aligned}
$$

donde $f(v)$ es definida como la función de acoplamiento; $\omega_{0}=\frac{E_{B}-E_{A}}{\hbar} ;$ donde $\mathrm{E}$ es la amplitud del campo electromagnético; $T_{1}$ y $T_{2}$ representan los tiempos de relajación longitudinal y transversal, respectivamente; 
$\rho_{D} \equiv \rho_{A A}-\rho_{B B}$ representan la diferencia de población entre los estados electrónicos adiabáticos; el superíndice (0) denota el valor de equilibrio en la diferencia poblacional en la ausencia de radiación. En términos del acoplamiento vibrónico, las cantidades de importancia que definen el Hamiltoniano de interacción radiación-materia, se definen de la forma:

$$
\begin{aligned}
& \mu_{B A}=\left(\sqrt{\frac{v^{2} e^{-S / 2}}{4 v^{2} e^{-S / 2}+\Delta^{2}}}\right)\left(m_{11}-m_{22}\right) \\
& -\Delta\left(\sqrt{\frac{e^{-S / 2}}{4 v^{2} e^{-S / 2}+\Delta^{2}}}\right) m_{21} \\
& d=\frac{\Delta e^{S / 2}\left(m_{11}-m_{22}\right)+4 v m_{21}}{\Delta^{2} e^{S / 2}+4 v^{2}} \sqrt{4 v^{2} e^{-S / 2}+\Delta^{2}} \\
& \operatorname{con} \Delta=E_{1 j}-E_{2 k}=-\omega_{0} \hbar \\
& \omega_{0}=\frac{1}{\hbar} \sqrt{\omega_{0}^{2} \hbar^{2}+4 v^{2} e^{-S / 2}}
\end{aligned}
$$

Es posible recuperar la dinámica del sistema (en su conjunto con la presencia del reservorio térmico e interactuando con la perturbación radiativa) en la representación diabática, haciendo la perturbación $H$ pequeña, por lo que $v \rightarrow 0$. Finalmente, las ecuaciones de Bloch ópticas convencionales en presencia del acoplamiento vibrónico, quedan definidas de la forma:

$$
\begin{aligned}
& \partial_{t} \rho_{A B}=\left(i \omega_{0}-\frac{1}{T_{2}}\right) \rho_{A B}+\frac{i}{\hbar}\left(\sqrt{\frac{v^{2} e^{-S / 2}}{4 v^{2} e^{-S / 2}+V_{0}^{2}}}\right) \\
& \times\left(\left(m_{a a}-m_{b b}\right)+\frac{V_{0}}{v} m_{b a}\right) \rho_{D} E \\
& -\frac{i}{\hbar}\left(\frac{-V_{0}\left(m_{a a}-m_{b b}\right)+4 v e^{-S / 2} m_{b a}}{\sqrt{4 v^{2} e^{-S / 2}+V_{0}^{2}}}\right) \rho_{A B} E \\
& \partial_{t} \rho_{D}=-\frac{1}{T_{1}}\left(\rho_{D}-\rho_{D}^{(0)}\right)-\frac{2 i}{\hbar}\left(\rho_{B A}-\rho_{A B}\right) \\
& \times\left(\sqrt{\left.\frac{v^{2} e^{-S / 2}}{4 v^{2} e^{-S / 2}+V_{0}^{2}}\right)\left(\left(m_{a a}-m_{b b}\right)+\frac{V_{0}}{v} m_{b a}\right)}\right. \\
& \left(\partial_{t} \rho_{B A}=\left(\partial_{t} \rho_{A B}\right)^{*}\right.
\end{aligned}
$$

Como puede observarse tanto en las coherencias como en las poblaciones, la dinámica queda definida en términos de los parámetros del acoplamiento, pero tiene información de las bases diabáticas como función de sus momentos dipolares. Tal como lo señalamos anteriormente, en la base diabática el sistema puede tener momentos dipolares permanentes nulos, y sin embargo el correspondiente momento dipolar en los estados adiabáticos no necesariamente son ceros. Esto obliga a que en el cálculo no se considere la aproximación de onda rotante y haya que incorporar en el cálculo todos aquellos términos de antiresonancia. Sin embargo, en la representación adiabática esta consideración ya no es determinante y por ende los resultados pueden concebirse sin la consideración de inclusión de términos que están fuera de resonancia.

\section{COMENTARIOS FINALES}

El modelo presentado en esta contribución representa una generalización o extensión de las ecuaciones ópticas de Bloch convencional en el régimen local de propagación. Normalmente en este tipo de ecuaciones locales no se consideran de forma explícita los momentos dipolares permanentes, ya que considerarlos hace que el tratamiento sea muy complejo y extenso por no poder hacer uso de la aproximación de la onda rotante. En un esquema perturbativo, incluir términos de anti-resonancia y estar fuera de la aproximación mencionada, requiere consideraciones muy particulares para evaluar las convergencias de los términos que escalan muy rápidamente, por ejemplo, cuando se trata interacción radiación-materia en el caso de mezcla de ondas tipo Rayleigh. Sin embargo, nuestra propuesta al convertir a la nueva base de estados adiabáticos, permite incorporar de forma natural los dipolos permanentes, los cuales son funciones de los dipolos en la base diabática, y estos últimos, pueden o no ser iguales a cero, pero el tratamiento no requiere condiciones de estar fuera de la aproximación de onda rotante. Esto hace, que nuestra propuesta, sea de utilidad para el estudio de los procesos de absorción de dos fotones, que normalmente se le estudia bajo consideraciones explicitas de momentos dipolares permanentes distintos de cero y tomando en cuenta los términos de anti-resonancia. Nuestro modelo puede ser aplicado a moléculas que presentan efectos del tipo Jahn-Teller o en los casos de estados excitados de dímeros. La restricción se cumplirá para los estados vibratorios casi degenerados en el llamado caso de acoplamiento débil.

\section{AGRADECIMIENTOS}

JLP agradece el financiamiento provisto por el Vicerectorado de investigación, Innovación y Vinculación de la Escuela Politécnica Nacional a través del proyecto PIIDFIS-2019-2. 


\section{REFERENCIAS}

Blum, K. (1981) Density Matrix Theory and Applications. Plenum Press

Charutz, D.M.; Baer, R.; Baer, M. (1997). A study of degenerate vibronic coupling effects on scattering processes: are resonances affected by degenerate vibronic coupling?. Chem. Phys. Lett. 265(6), 629637. 2614(96)01494-7.

Di Bartolo B.; Goldberg, V. (1980) Radiationless Processes. Springer

Domcke, W.; Stock, G. (1997). Theory of ultrafast nonadiabatic excited-state processes and their spectroscopic detection in real-time. Adv. Chem. Phys. 100, 1-169

García-Sucre, M.; Goychman, F.; Lefebvre, R. (1970). Adiabatic corrections in a simple model of twointeracting electronic-potential curves. Phys. Rev. A, 2 (5), 1738-1745. https://doi.org/10.1103/PhysRevA.2.1738

Mebel, A.; Hayashi, M.; Lin, S.H. (1997). Ab-initio calculations of vibronic coupling. Application to symmetry-forbidden vibronic spectra and internal conversion in ethylene. Chem. Phys. Lett. 274 (1$3)$ 281-292. https://doi.org/10.1016/S00092614(97)00654-4

Mita, T.; Uchiyama, M.; Soto, Y. (2020). Catalytic intramolecular coupling of Ketoalkenes by Allylic $\mathrm{c}\left(\mathrm{sp}^{3}\right)-\mathrm{H}$ bond Cleavage: Synthesis of Five- and six-membered Carbocyclic compounds. Advanced Synthesis and catalysis, 362 (6), 1275-1280. https://doi.org/10.1002/adsc.202000184

Pakhomov, A.; Ekbundit, S.; Lin, C.H.; Alden, R.G.; Lin, S. H. (1995). Effect of intermolecular vibronic coupling on non-radiative transitions and optical absorption of dimmers. J. Lummin. 63, 129-136

Slivnev, V.V.; Dimenov, O.A.; Girichev, G.V. (2020). Jahn-Teller effect versus spin-orbit coupling: The structure of the free molybdenum pentafluoride molecule. J. of Molecular Structure, 1199, 126884 (11 pages). 10.1016/j.molstruc.2019.126884

Thoss, M.; Miller, W.H.; Stock, G. (2000). Semiclassical description of non-adiabatic quantum dynamics: Application to the $\mathrm{S}_{1}-\mathrm{S}_{2}$ conical intersection in pyrazine. J. Chem. Phys. 112, 10282-10292. https://doi.org/10.1063/1.481668
Witkowsky, A.; Moffitt, W. (1960). Electronic spectra of dimmers: derivation of the fundamental vibronic equation. J. Chem. Phys. 33, 872-875. https://doi.org/10.1063/1.1731278

Zhao, L.; Tao, Z.; Pavosevic F.; Wildman, A.; HammesSchiffer, S.; Li, X. (2020). Real-Time Timedependent nuclear-electronic orbital approach: Dynamics beyond the Born-Oppenheimer approximation. The Journal of Phys. Chem. Lett. 11(10), 4052-4058. https://doi.org/10.1021/acs.jpclett.0c00701

\section{BIOGRAFÍAS}

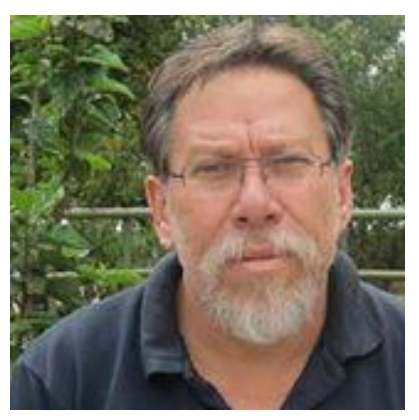

José Luis Paz. Licenciado en Química (1983), M. Sc en Química (1985) y Doctorado en Química (Universidad Central de Venezuela, 1988). Postdoctorado en Optica Cuántica UNAM. Profesor Jubilado Emérito (2014)-de la Universidad Simón Bolívar, Caracas, Venezuela. Individuo de Número de la Academia de Ciencias Físicas, Matemáticas y Naturales, Venezuela (2009). Investigador Senior Prometeo (20142017) Ecuador. Profesor Invitado adscrito al departamento de Física, EPN. Línea de investigación en Óptica No lineal y Óptica Cuántica. https://www.researchgate.net/profile/Jose_Paz3/research http://ORCID/0000-0002-6177-7919

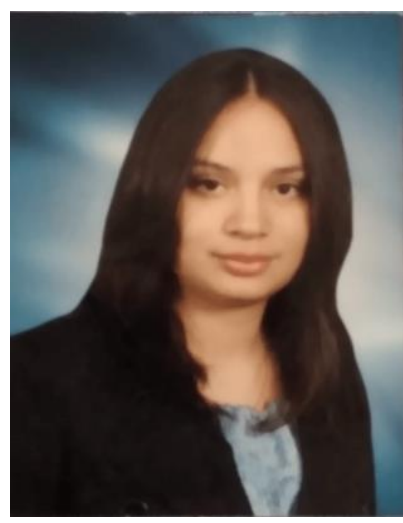

Eleana de los Ángeles Ruiz Hinojosa. Física graduada en la Escuela Politécnica Nacional (EPN). Realizó su tesis de pregrado sobre óptica no lineal titulada "Efectos del acoplamiento intramolecular en el estudio de las respuestas ópticas no lineales en colorantes orgánicos en la señal de Mezcla de Cuatro Ondas". Tiene experiencia en estudios de espectroscopia, exfoliación de grafeno y la deposición de películas delgadas para la producción de celdas solares, además del manejo de varios lenguajes de programación, tales como: Fortran, Matlab, Mathematica, Python, C++. Sus intereses de investigación teórica y experimental son óptica no lineal y mecánica cuántica. https://orcid.org/0000-0002-7063-3466 


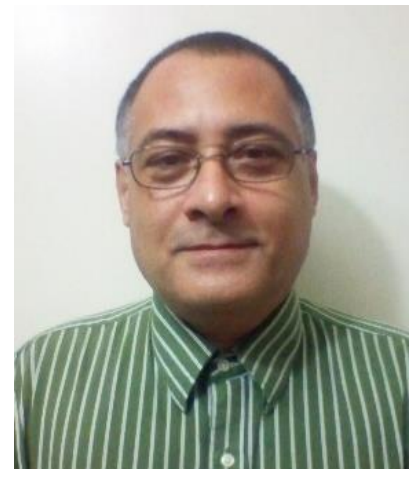

Ysaias J. Alvarado. Licenciado en Química (1992) en la Universidad del Zulia, Venezuela. Doctorado en Química (1996) en el Instituto Venezolano de Investigaciones Científicas (IVIC) bajo la supervisión del Dr. Roberto Sánchez Delgado. Profesor Titular de la Facultad Experimental de Ciencias (FEC) de la Universidad del Zulia (LUZ). Fundador del Laboratorio de Electrónica Molecular en el Departamento de Química en dicha Universidad. Miembro fundador del Doctorado en Química en la FEC. Investigador Asociado Titular del Instituto Venezolano de Investigaciones Científicas (IVIC). Fundador del Laboratorio de Caracterización Molecular y Biomolecular en el Centro de Investigación y Tecnología de Materiales (CITeMA) del IVIC. Jefe del Centro de Investigación y Tecnología de Materiales (CITeMA) del IVIC. https://orcid.org/0000-0002-2709-409X

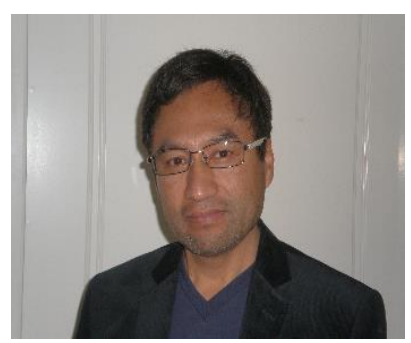

Luis Lascano Lascano. Doctorado en Física de Materiales, Universidad Autónoma de Madrid e Instituto de Cerámica y Vidrio, España. Físico, Escuela Politécnica Nacional (EPN), Quito. Profesor Principal del Departamento de Física de la EPN. Miembro del Grupo de Investigación de Materiales Electrónicos y Magnéticos de dicho departamento. Las líneas de investigación que desarrolla son: Estudios teórico y experimental de Materiales Electrocerámicos Ferroeléctricos, y estudios teóricos en Óptica no lineal. https://orcid.org/0000-0001-7588-0194

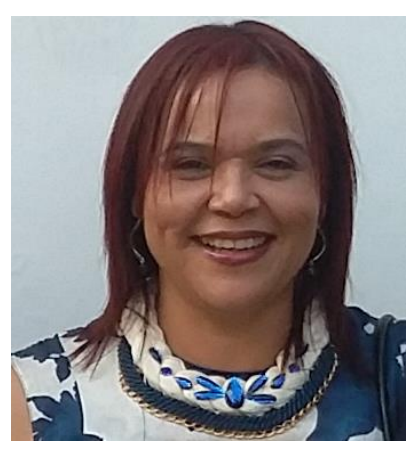

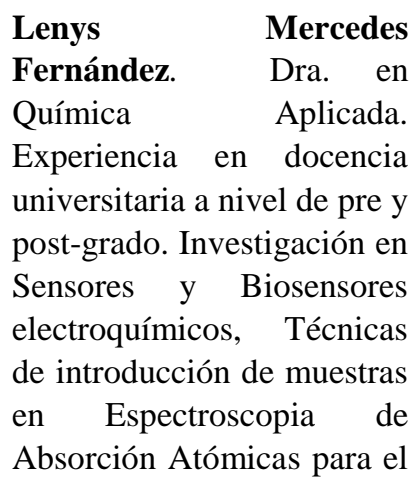
análisis de metales pesados en muestras de ambientales y/o de alimentos. Revisora de libros y artículos en revistas científicas indexadas. Autora de siete (7) libros, sesenta y dos (72) artículos científicos, tres (03) capítulos de libros, noventa ocho (98) asistencias a congresos en su área de especialización y dos (02) premios por los mejores trabajos de investigación a nivel doctoral (ULA-Venezuela). Actualmente, profesora en la Escuela de Ciencias Química de la Pontificia Universidad Católica del Ecuador. ORCID: 0000-0001-6720-6343

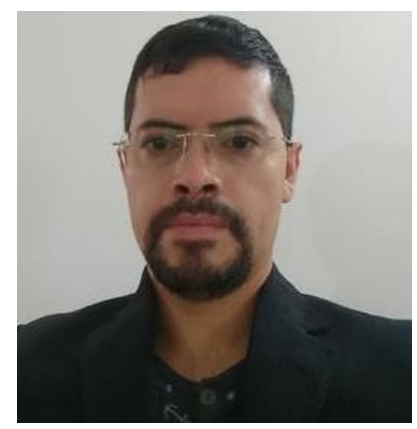

Patricio Javier

Espinoza-Montero.

Químico por la Escuela de Química de la Universidad Central del Ecuador (2007); Maestro en Ciencias por la Universidad Nacional Autónoma de México (UNAM) (2009); Doctor en Ciencias por UNAM (2014). Experto en Polímeros Conductores, Procesos de Oxidación Avanzados para el tratamiento de aguas residuales (Electrooxidación, Fotocatálisis, Fotoelectrocatálisis), Sensores, Biosensores, Inmunosensores, Electrospinning. Estancia de investigación (6 meses), Universidad Iberoamericana de México-Campus Santa Fe (2012). En 2013 realizó estancia de investigación de 6 meses en la Michigan State University-USA (Electroquímica Analítica). Destacan 42 artículos científicos publicado y dos capítulos de libro. Profesor en Pontificia Universidad Católica del Ecuador, Escuela de Ciencias Químicas. ORCID: 0000-0003-0592-8652

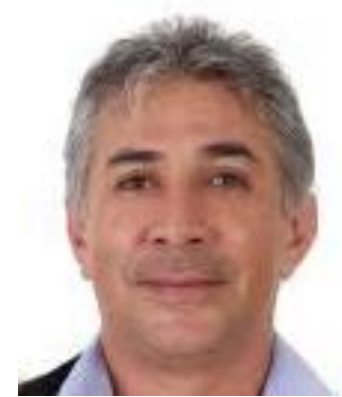

César Costa-Vera. Físico (1992), graduado en la Escuela Politécnica Nacional, Doctorado en Física de Iones (1999) en programa cooperativo entre la Escuela Politécnica Nacional y la Universidad de Uppsala en Suecia. Posdoctorado (Beca Georg Forster), Fundación Alexander von Humboldt, en Universidad de Giessen, Alemania. Miembro de la Academia de Ciencias del Ecuador. Líder del Grupo Mass Spectrometry \& Optical Spectroscopy, EPN. Trabaja en óptica, fotónica, láseres y nanoespectroscopía. Profesor Principal del Departamento de Física de la EPN. https://www.scopus.com/authid/detail.uri?authorId=15041 572100

https://orcid.org/0000-0001-5242-1892 
